\title{
Corrigendum: Quantitative evaluation of gender bias in astronomical publications from
} citation counts

Neven Caplar, Sandro Tacchella and Simon Birrer

Nature Astronomy 1, 0141 (2017); published 26 May 2017; corrected 19 June 2017.

In the version of this Letter originally published, the author's name in ref. 11 was incorrect and should have read 'Reid, I. N.' 